\section{Impacto da internação na prática do aleitamento materno em hospital pediátrico de Salvador, Bahia, Brasil}

\author{
Impact of hospitalization on breastfeeding \\ practices in a pediatric hospital in \\ Salvador, Bahia State, Brazil
}

\section{${ }^{1}$ Faculdade de Medicina da Bahia, Universidade Federal da Bahia, Salvador, Brasil. \\ Correspondência \\ E. L. Souza \\ Departamento de Pediatria, Faculdade de Medicina da Bahia, Universidade Federal da Bahia. \\ Av. Paulo VI 2200, apto. 104 Salvador, BA 41810-001, \\ Brasil. \\ ednalu@ufba.br}

\section{Abstract}

This cross-sectional study enrolled 97 inpatients at a teaching hospital in Salvador, Bahia, Brazil, to determine breastfeeding prevalence in infants less than 4 months of age hospitalized due to respiratory infection, and to evaluate the impact of hospitalization on breastfeeding. Patients' mothers were interviewed, and a standardized questionnaire was completed. After hospital discharge, medical records were reviewed and information on the infant's feeding practices during hospitalization was recorded. Exclusive breastfeeding was observed in $57.1 \%$ of patients, but it was interrupted in $35.4 \%$, with the introduction of infant formula during hospitalization. Mean duration was not associated with the introduction of complementary feeding in the hospital. In the bivariate analysis, early interruption of exclusive breastfeeding was associated with higher maternal schooling and lower family income. Prevalence of exclusive breastfeeding was low. Hospitalization contributed to early interruption of exclusive breastfeeding in $35.4 \%$ of infants, possibly due to inadequate hospital infrastructure and insufficient support from health professionals to maintain exclusive breastfeeding.

Breast Feeding; Weaning; Hospitalization; Infant; Respiratory Tract Infections
Edna Lúcia Souza ${ }^{1}$

Luciana Rodrigues Silva 1

Ana Carolina Souza Sá 1

Clara Maia Bastos 1

Andrea Borges Diniz ${ }^{1}$

Carlos Maurício Cardeal Mendes 1

\section{Introdução}

A Organização Mundial da Saúde (OMS) recomenda que todas as crianças recebam aleitamento materno exclusivo até os seis meses de vida e que, após esse período, continuem sendo amamentadas, até os dois anos ou mais, juntamente com a utilização de alimentos complementares 1 .

Diversas evidências apontam para os efeitos benéficos do aleitamento materno para a criança, na prevenção de doenças a curto e a longo prazo, além daqueles de ordem nutricional, psicológica, afetiva, social e econômica 1,2 . Apesar disso, a prevalência de aleitamento materno exclusivo ainda é baixa no Brasil. Dados do Ministério da Saúde 3 evidenciaram que, em 1999, $88 \%$ dos lactentes de 0 a 30 dias de vida eram amamentados, mas apenas $53,1 \%$ o faziam de forma exclusiva. Na Região Nordeste, a porcentagem de crianças amamentadas foi ainda menor $(86,7 \%)$, embora a porcentagem de aleitamento exclusivo tenha sido mais elevada $(55,4 \%)$. Esse mesmo estudo demonstrou que, em Salvador, Bahia, 85,5\% dos lactentes de 0 a 30 dias de idade eram amamentados, mas apenas $43 \%$ estavam em aleitamento materno exclusivo. Os dados revelaram outro ponto alarmante: apenas 9,7\% das crianças brasileiras atingiam os seis meses de vida em aleitamento materno exclusivo. No Nordeste, a porcentagem foi de $10,7 \%$ e, em Salvador, $6,7 \%$. Por outro lado, os dados da cidade de Feira 
de Santana, Bahia, que dispõe de um amplo programa de incentivo à amamentação, apresentaram melhores indicadores, quando comparados a Salvador e outras cidades da Região Nordeste, observando-se que $69,2 \%$ das crianças menores de um ano ainda eram amamentadas, enquanto $38,5 \%$ daquelas menores de sete meses faziam aleitamento materno exclusivo 4 .

O êxito na prática do aleitamento materno relaciona-se com as características biológicas da criança e da mãe, bem como aos fatores sócioculturais e às práticas desenvolvidas nos serviços de saúde 5 . Nesse sentido, a criação do programa Iniciativa Hospital Amigo da Criança (IHAC), em 1992, teve como principal objetivo a capacitação de profissionais de saúde e dos próprios estabelecimentos de saúde como maternidades, para o fornecimento de informações corretas às mães, durante a internação, sobre a amamentação e para a adoção de práticas e rotinas favoráveis ao aleitamento materno. Com isso, enfatizou-se a grande importância dos serviços de atendimento à saúde na proteção, promoção e apoio à prática do aleitamento materno ${ }^{6}$. Entretanto, o incentivo à prática da amamentação não pode se restringir ao ambiente das maternidades, devendo ser estendido a todas as instituições de saúde que atendem crianças, inclusive às unidades de internação hospitalar, no intuito não apenas de promover a iniciação dessa prática, mas também zelar pela sua manutenção. A literatura é escassa sobre a influência do internamento hospitalar pós-natal sobre a prática da amamentação.

Este trabalho teve como objetivos determinar a prevalência de aleitamento materno entre as crianças menores de quatro meses, internadas por infecção respiratória, em um hospital universitário da cidade de Salvador, e descrever a influência da internação sobre a prática do aleitamento materno.

\section{Casuística, material e métodos}

Este foi um estudo do tipo corte transversal, realizado durante um período de vinte meses. A população estudada integra uma investigação de infecção respiratória em lactentes. Foram incluídos na pesquisa todos os pacientes internados na Unidade de Pequenos Lactentes (UPL) do Centro Pediátrico Professor Hosannah de Oliveira (CPPHO) da Universidade Federal da Bahia (UFBA), entre julho de 2004 e fevereiro de 2006, com diagnóstico de pneumonia ou bronquiolite. A UPL caracteriza-se por prestar a assistência a lactentes de até quatro meses de idade.

A pneumonia foi definida por tosse e/ou dificuldade para respirar, associada a alterações radiológicas (condensação alveolar e/ou broncopneumonia e/ou pneumatocele e/ou derrame pleural e/ou abscesso). A bronquiolite foi definida pela ocorrência de tosse, taquipnéia, ausculta de crépitos e/ou sibilância, associadas ou não a alterações radiológicas, como sinais de hiperinsuflação e/ou atelectasias.

As mães ou responsáveis pelas crianças foram entrevistados por estudantes de medicina da Faculdade de Medicina da Bahia (FAMEB) da UFBA, previamente treinadas pelo pesquisador principal. Foi aplicado um questionário para coleta de informações sobre: características demográficas, realização do atendimento pré-natal, perda prematura de líquido, condições do parto e do nascimento e o quadro clínico da criança. As informações referentes ao aleitamento materno, introdução de outros alimentos e desmame foram pesquisadas através da ficha de anamnese padronizada do hospital, onde constam as informações sobre a alimentação. Após a alta hospitalar, o prontuário foi revisado, registrandose as informações sobre as práticas alimentares durante a internação.

$\mathrm{O}$ aleitamento materno foi definido como exclusivo quando a criança só recebia o leite materno e nenhum outro sólido ou líquido, com exceção de vitaminas ou medicamentos 1 .

Todos os dados obtidos foram registrados em questionário padrão e armazenados em um banco de dados no programa Microsoft Access (Microsoft Corp., Estados Unidos). Para análise estatística, foi utilizado o programa $\mathrm{R}$ (The R Foundation for Statistical Computing, Viena, Áustria; http://www.r-project.org). A análise descritiva consistiu no cálculo das freqüências simples e relativas das variáveis estudadas com o objetivo de conhecer o perfil das mães e das crianças internadas. Com a intenção de verificar possíveis associações entre co-variáveis e a interrupção da amamentação exclusiva ou desmame, efetuou-se análise bivariada, utilizando-se o teste de Wilcoxon e a correlação de Sperman, em função da natureza das variáveis subjacentes. O teste de McNemar foi utilizado para avaliar a significância da mudança antes, e após a internação, em relação à manutenção ou não do aleitamento materno exclusivo. Foram considerados estatisticamente significantes os resultados que tiveram a probabilidade de erro tipo 1 menor que $5 \%(\mathrm{p}<0,05)$.

Este estudo foi aprovado pelo Comitê de Ética em Pesquisa da Maternidade Climério de Oliveira da UFBA (parecer no. 23/2004). Todos os responsáveis pelos pacientes participantes do estudo assinaram o Termo de Consentimento Livre e Esclarecido. 


\section{Resultados}

Entre julho de 2004 e fevereiro de 2006, foram internadas 99 crianças menores de quatro meses de idade e duas crianças com idade superior a quatro meses, com diagnóstico de pneumonia ou bronquiolite, na UPL do CPPHO. Noventa e sete lactentes foram incluídos no estudo. Quatro crianças foram excluídas: duas por extravio dos prontuários e duas por impossibilidade de obtenção de dados referentes ao aleitamento materno.

Sessenta e sete $(69,1 \%)$ crianças eram do sexo masculino e 30 (30,9\%), do sexo feminino. A idade dos lactentes variou de 8 a 169 dias, com uma média de 52,5 dias ( $\mathrm{DP}=24,74)$ e mediana de 52 dias. A maioria dos lactentes procedia de bairros periféricos de Salvador ou de municípios próximos. A duração do internamento variou de 2 a 248 dias, com uma média de 16,1 e mediana de 9 dias. A Tabela 1 apresenta as características demográficas e as condições do nascimento destes lactentes.

O diagnóstico que mais freqüentemente motivou o internamento foi a bronquiolite, com 62 casos (63,9\%). Dessas crianças, 19 tinham diagnóstico associado de pneumonia. A média de freqüência respiratória dos pacientes à admissão foi $59,3 \pm 12,9$. Trinta lactentes (30,9\%) necessitaram de oxigenioterapia, sendo que $60 \%$ desses o fizeram pelo período máximo de três dias.

Setenta e cinco $(77,3 \%)$ pacientes apresentavam comorbidades. Desses, 51 (68\%) apresentavam mais de uma comorbidade. As principais comorbidades foram: anemia (28\%), monilíase oral $(26,7 \%)$ e hérnias umbilical ou inguinal $(25,3 \%)$. Treze $(13,4 \%)$ crianças apresentavam atraso vacinal.

Do total de 97 crianças incluídas no estudo, $82(85,4 \%)$ mães realizaram acompanhamento pré-natal, sendo que apenas $8(8,5 \%)$ tiveram nove ou mais consultas nesse período.

Entre os lactentes estudados, 84 (86,6\%) estavam em aleitamento materno na admissão hospitalar. Oito $(8,2 \%)$ crianças nunca haviam sido amamentadas e cinco já haviam sido desmamadas antes da hospitalização. Entre as crianças que estavam em aleitamento materno, 48 (57,1\%) faziam aleitamento materno exclusivo no momento da internação, enquanto 36 já recebiam leite ou outros alimentos. Entre as 48 crianças com aleitamento materno exclusivo, 17 (35,4\%) tiveram o aleitamento exclusivo interrompido pela introdução de fórmula láctea no hospital, não se incluindo aqui aquelas que receberam apenas outros líquidos. Enquanto que, entre as 36 crianças que já não estavam em aleitamento exclusivo, nenhuma reverteu esse processo. A associação entre internação hospitalar e interrupção do aleitamento materno exclusivo foi estatisticamente significante ( $\mathrm{p}=0,00003738)$ (Tabela 2).

Trinta e seis $(42,9 \%)$ lactentes já recebiam outros leites ou outros alimentos no início da internação. Vinte e nove $(29,9 \%)$ crianças interromperam o aleitamento materno exclusivo no primeiro mês de vida, incluindo aquelas que o fizeram durante a hospitalização.

Trinta e uma crianças permaneceram em aleitamento materno exclusivo até a alta e $75 \%$ dessas tinham menos de dois meses de vida. Esses pacientes tinham freqüência respiratória média à admissão de 66,1 $\pm 10,0$, enquanto o restante das crianças apresentava freqüência respiratória média de $60 \pm 13,9$. As características dessa subpopulação podem ser visualizadas na Tabela 3 .

Incluindo-se as oito crianças que nunca foram amamentadas, 15 já estavam desmamadas ao final da hospitalização. Dessas, duas foram desmamadas no período de internamento.

Setenta e sete lactentes realizaram investigação sorológica para infecção congênita ou perinatal, dos quais 12 (15,6\%) obtiveram algum resultado positivo. Oito crianças tiveram a imunoglobulina M positiva para o citomegalovírus e duas foram positivas para o HIV. Sessenta e sete crianças realizaram a sorologia para Chlamydia trachomatis e $9(13,4 \%)$ tiveram resultado positivo.

Não foi observada associação entre a idade do desmame e a realização de pré-natal $(\mathrm{p}=$ $0,8705)$. A idade do desmame não se associou ao número de consultas pré-natais (correlação de Spearman = 0,0589; $\mathrm{p}=0$,8557). Também não houve associação entre a idade do desmame e a idade materna (correlação de Spearman $=0,242$; $\mathrm{p}=0,3856$ ). Houve uma associação significante entre a idade do desmame e a escolaridade materna, de tal forma que, quanto maior o grau de escolaridade materna, mais tardio o desmame ( $p=0,0442)$, entretanto, essa diferença não foi tão importante, de tal forma que a diferença entre as medianas nos dois grupos de mães com maior e menor escolaridade foi de apenas um dia. Também houve uma associação entre menor renda familiar e o desmame mais tardio, mas neste caso, sem significância estatística ( $\mathrm{p}=0,2481$ ).

A introdução de fórmulas lácteas durante a hospitalização não se associou à duração da internação. A mediana da internação do grupo que teve a introdução de fórmulas lácteas no hospital foi 9 dias, enquanto foi 12 dias para as crianças que não introduziram fórmulas lácteas. Entretanto, a avaliação do conjunto das distribuições não demonstrou diferenças (Tabela 4). A idade da criança, à época da introdução de fórmulas lácteas ou outros alimentos, não esteve associa- 
Características demográficas e condições de nascimento dos lactentes internados por infecção respiratória em hospital pediátrico de Salvador, Bahia, Brasil, 2007.

\begin{tabular}{|c|c|c|}
\hline Variável & $\mathrm{n}$ & $\%$ \\
\hline \multicolumn{3}{|l|}{ Idade materna (anos) * } \\
\hline Média & $24,26(\mathrm{DP}=5,9)$ & - \\
\hline Mediana & 23 & - \\
\hline Até 20 & 25 & 25,8 \\
\hline Maiores de 20 & 71 & 73,2 \\
\hline \multicolumn{3}{|l|}{ Escolaridade materna } \\
\hline Sem escolaridade & 7 & 7,2 \\
\hline Alfabetizada & 1 & 1,0 \\
\hline 1ㅇ grau completo & 12 & 12,3 \\
\hline 1 o grau incompleto & 38 & 39,2 \\
\hline 2o grau completo ou incompleto & 32 & 32,9 \\
\hline Superior completo ou incompleto & 2 & 2,1 \\
\hline Sem informação & 5 & 5,2 \\
\hline \multicolumn{3}{|l|}{ Renda familiar (salários mínimos) } \\
\hline Sem renda fixa & 40 & 41,2 \\
\hline Até 1 & 25 & 25,8 \\
\hline 2 & 18 & 18,5 \\
\hline 3 ou mais & 9 & 9,3 \\
\hline Sem informação & 5 & 5,1 \\
\hline Realização de pré-natal & 82 & 84,5 \\
\hline \multicolumn{3}{|l|}{ Tipo do parto } \\
\hline Vaginal & 69 & 71,1 \\
\hline Abdominal & 27 & 27,8 \\
\hline Fórceps & 1 & 1,0 \\
\hline \multicolumn{3}{|l|}{ Idade gestacional } \\
\hline A termo & 76 & 77,6 \\
\hline \multicolumn{3}{|l|}{ Peso ao nascer (gramas) * } \\
\hline$\geq 2.500$ & 79 & 82,2 \\
\hline$<2.500$ & 16 & 17,7 \\
\hline
\end{tabular}

DP: desvio-padrão.

* Uma informação perdida

da à idade materna (correlação de Spearman = $-0,0853 ; p=0,5434$ ). A idade da criança a época da introdução de fórmulas lácteas ou outros alimentos demonstrou associação com a escolaridade materna $(\mathrm{p}<0,001)$. Mães com escolaridade até o $1^{\circ}$ grau introduziram outros alimentos, em média, com 29,8 dias e mediana de 30 dias, enquanto aquelas com escolaridade igual ou superior ao $2^{\circ}$ grau, interromperam o aleitamento materno exclusivo, em média, com 21,7 dias e mediana de 20 dias.

A idade da criança, à época da introdução de fórmulas lácteas ou outros alimentos, esteve relacionada à renda familiar $(\mathrm{p}<0,001)$, de tal forma que, quanto menor a renda, mais precocemente o aleitamento materno exclusivo foi interrom- pido. Famílias com renda inferior a um salário mínimo introduziram outras fórmulas lácteas ou alimentos na dieta de seus filhos, em média, aos 26 dias de vida e com mediana de 22 dias. Por outro lado, as famílias com renda entre um e dois salários mínimos mantiveram o aleitamento materno exclusivo até 27 dias de vida, em média, e com uma mediana de 23 dias. 


\begin{tabular}{lccc}
\hline & & \multicolumn{2}{c}{ Alta } \\
\hline Admissão & & AME & AM \\
& $\mathrm{N}(\%)$ & $\mathrm{N}(\%)$ & $\mathrm{N}(\%)$ \\
AME & $48(57,1)$ & $31(64,6)$ & $17(35,4)$ \\
AM & $36(42,9)$ & $0(0,0)$ & $34(94,4) * *$ \\
Total & $84(100,0)$ & $31(37,8)$ & $51(62,2)$ \\
\hline
\end{tabular}

* Análise estatística: teste de McNemar = 17; g.l. = 1; $p=0,00003738$;

** Duas crianças foram desmamadas durante a hospitalização.

\section{Discussão}

Cabe destacar que esta amostra estudada corresponde a uma população selecionada de crianças internadas por infecção respiratória e compreende, principalmente, famílias com baixas condições sócio-econômicas, que representam a grande maioria da população brasileira e de Salvador. Entretanto, os resultados não poderão ser extrapolados para outras populações. É importante esclarecer, também, que os dados referentes à amamentação foram coletados de forma retrospectiva, fato que apresenta limitações inerentes à metodologia do estudo.

Os resultados revelaram baixa prevalência $(49,5 \%)$ de aleitamento materno exclusivo, particularmente se considerarmos a média de idade das crianças. Os dados sugerem que, em Salvador, muitos lactentes têm introdução precoce de outros alimentos, muitos deles, de modo inadequado, já no primeiro mês de vida. Por outro lado, comparando-se aos dados do Ministério da Saúde de 1999 e ao estudo de Oliveira et al. 7, em Salvador, foi observada uma maior prevalência de aleitamento materno exclusivo, ressaltandose que este estudo avaliou uma amostra selecionada de crianças, ao contrário dos dois citados, que foram de base populacional e, portanto, são mais representativos.

A constatação de que $17,5 \%$ das crianças incluídas nesse estudo tiveram o aleitamento materno exclusivo interrompido pela introdução de fórmulas lácteas durante a internação revelou-se alarmante e surpreendente. Os dados da freqüência respiratória média das crianças à internação, bem como a utilização de oxigênio foram semelhantes entre aquelas que se mantiveram em aleitamento materno exclusivo e as demais. Além disso, a observação de que a interrupção do aleitamento materno exclusivo não se associou com a duração da internação, ou seja, mesmo crianças que permaneceram internadas por períodos curtos tiveram o aleitamento materno exclusivo suspenso, indica que a introdução de fórmulas lácteas não se relacionou com a gravidade do quadro clínico do lactente, e sugere que reflita muito mais uma prática rotineira do serviço do que as dificuldades inerentes à hospitalização, relacionadas às crianças e as suas mães, como bem apontaram Serra \& Scochi 8 , em estudo qualitativo, conduzido numa unidade de terapia intensiva neonatal, em Cuiabá, Mato Grosso. Os autores descreveram alguns obstáculos significativos à prática do aleitamento materno dos lactentes internados e constataram a falta de rotinas e práticas de incentivo ao aleitamento, além das dificuldades de ordem física, como o desconforto das acomodações para as mães e o fornecimento de poucas refeições ao dia. O CPPHO, hospital deste estudo, não dispõe de banco de leite. As mães são acomodadas em cadeiras plásticas reclináveis, com pouco conforto, sobretudo quando a hospitalização é prolongada. As lactantes, cujos filhos estão sob aleitamento materno exclusivo, recebem até cinco refeições diárias, enquanto acompanhantes. Deve-se ressaltar que muitas dessas mães têm domicílio distante do hospital e que, muitas vezes, têm outras crianças que necessitam de sua atenção, o que dificulta a sua permanência em tempo integral no CPPHO, bem como sua locomoção. Pode-se, também, levantar a hipótese da falta de apoio dos companheiros e demais familiares para a manutenção da amamentação.

Apesar de a unidade prestar assistência a lactentes jovens, não existem normas que estimulem a prática do aleitamento materno e previna a sua interrupção. Cabe destacar, também, que 
Características das crianças que permaneceram em aleitamento materno exclusivo durante a internação em hospital pediátrico de Salvador, Bahia, Brasil, 2007.

\begin{tabular}{|c|c|c|}
\hline Variáveis & $\mathrm{n}$ & $\%$ \\
\hline \multicolumn{3}{|l|}{ Sexo } \\
\hline Masculino & 24 & 77,40 \\
\hline Feminino & 7 & 22,60 \\
\hline \multicolumn{3}{|l|}{ Idade do lactente (dias) } \\
\hline Até 30 & 6 & 19,40 \\
\hline 31 a 60 & 17 & 54,80 \\
\hline 61 a 90 & 8 & 25,80 \\
\hline Mais de 90 & - & - \\
\hline \multicolumn{3}{|l|}{ Idade materna (anos) } \\
\hline Até 20 & 7 & 22,60 \\
\hline 21 a 30 & 18 & 58,00 \\
\hline 31 a 40 & 6 & 19,40 \\
\hline Mais de 40 & - & - \\
\hline \multicolumn{3}{|l|}{ Renda familiar (salários mínimos) } \\
\hline Sem renda fixa & 11 & 35,50 \\
\hline 1 & 9 & 29,00 \\
\hline 2 & 7 & 22,60 \\
\hline 3 ou mais & 3 & 9,70 \\
\hline Sem informação & 1 & 3,20 \\
\hline \multicolumn{3}{|l|}{ Escolaridade materna } \\
\hline Sem escolaridade & 2 & 6,50 \\
\hline 1ㅇ grau completo e incompleto & 16 & 51,60 \\
\hline 2o grau completo e incompleto & 10 & 32,30 \\
\hline Superior completo e incompleto & 2 & 6,45 \\
\hline Sem informação & 1 & 3,20 \\
\hline \multicolumn{3}{|l|}{ Realização de pré-natal } \\
\hline Sim & 28 & 90,30 \\
\hline Não & 3 & 9,70 \\
\hline \multicolumn{3}{|l|}{ Tipo de parto } \\
\hline Vaginal & 21 & 67,70 \\
\hline Abdominal & 9 & 29,00 \\
\hline Fórceps & 1 & 3,20 \\
\hline \multicolumn{3}{|l|}{ Uso de oxigênio (dias) } \\
\hline Não & 25 & 80,60 \\
\hline 1 & 3 & 9,70 \\
\hline 3 & 2 & 6,50 \\
\hline 6 & 1 & 3,20 \\
\hline \multicolumn{3}{|l|}{ Duração da internação hospitalar (dias) } \\
\hline Até 7 & 17 & 54,80 \\
\hline 8 a 14 & 10 & 32,30 \\
\hline Mais de 14 & 4 & 12,90 \\
\hline
\end{tabular}

Nota: média da freqüência respiratória à internação: $61,6 \pm 10,0$. 
Duração da internação hospitalar, em dias, nos grupos de crianças que iniciaram e que não iniciaram fórmula láctea no hospital.

\begin{tabular}{lccccc}
\hline & Quartil 5 & Quartil 25 & Quartil 50 & Quartil 75 & Quartil 95 \\
\hline Não iniciaram fórmula láctea $(n=49)$ & 4 & 8 & 12 & 16 & 30,2 \\
Iniciaram fórmula láctea $(n=17)$ & 4,6 & 8 & 9 & 15 & 134 \\
\hline
\end{tabular}

se trata de um hospital escola, contribuindo para a formação de diversos profissionais da área de saúde como médicos, enfermeiros, nutricionistas e fisioterapeutas, portanto os dados demonstrados nesse estudo apresentam uma grande relevância, uma vez que se esperava que houvesse uma rotina que estimulasse a amamentação, contribuindo, também, na formação de profissionais preparados para assistir e estimular a lactação.

Além das limitações de ordem física, já relatadas, esses resultados revelaram que os profissionais de saúde, envolvidos na assistência a essas crianças, não estão preparados no sentido de orientar as mães e estimulá-las, adequadamente, para a manutenção do aleitamento, nem a buscarem, quando necessário, formas alternativas, como a ordenha do leite, por exemplo, ou solucionarem outros problemas e dúvidas comuns, relacionados com a prática do aleitamento. $\mathrm{O}$ estudo de Quattrone et al. ${ }^{9}$ avaliou o impacto da internação na prática da amamentação entre 108 lactentes, menores de seis meses, em um hospital de Buenos Aires, Argentina. Os autores observaram que $60 \%$ dos lactentes que eram amamentados no início da internação foram desmamados até a alta, dos quais $42 \%$ o fizeram apenas pela hospitalização e não por causas médicas, além de $17 \%$ terem relatado "hipogalactia". Esses dados, ainda mais relevantes que os nossos, demonstram que, possivelmente, as equipes de saúde têm falhado no incentivo à amamentação.

A IHAC tem demonstrado francos benefícios à amamentação, mas está limitada a maternidades. Desconhecemos qualquer iniciativa semelhante para hospitais pediátricos, que não são maternidades, e assistem crianças nos primeiros anos de vida. Em um estudo realizado em Salvador, Silva et al. 10 avaliaram o conhecimento materno sobre aleitamento e observaram o alto grau de desinformação das mães sobre o tema, destacando o papel dos profissionais de saúde no esclarecimento de dúvidas, especialmente durante o pré-natal. O estudo de Vannuchi et al. 11 demonstrou um impacto positivo da IHAC mesmo entre crianças que foram internadas no período neonatal. Esses achados enfatizam a importância da capacitação sistemática de profissionais e dos serviços da saúde, contribuindo para o êxito da prática de aleitamento materno entre as crianças hospitalizadas. O momento da hospitalização pode representar uma oportunidade para sistematizar várias condutas adequadas para o futuro das crianças, tais como: aleitamento materno, nutrição sadia, imunizações, prevenção de acidentes e de infecções, através de reuniões entre os profissionais de saúde e as mães.

Neste estudo, foi demonstrada uma associação entre o nível sócio-econômico da família e a introdução de alimentos complementares. Os lactentes nascidos em famílias de baixa renda tiveram o aleitamento exclusivo interrompido mais precocemente. Esses dados concordam com o trabalho de Mascarenhas et al. 12 que avaliou lactentes entre um e três meses de idade, na cidade de Pelotas, Rio Grande do Sul, e observou uma associação entre a baixa renda familiar e a interrupção do aleitamento exclusivo antes dos três meses de idade. Oliveira et al. 7 estudaram os fatores associados à interrupção precoce do aleitamento exclusivo ou predominante em crianças menores de 24 meses de idade, da cidade de Salvador, e observaram que as condições precárias de vida estavam associadas à introdução de alimento complementar e desmame precoces. Por outro lado, em um trabalho realizado na cidade de Feira de Santana, entre crianças menores de um ano de idade, Vieira et al. ${ }^{4}$ encontraram maior prevalência de aleitamento materno entre as crianças nascidas em famílias de baixa renda. Ressalte-se que essa cidade apresenta um trabalho sistemático de incentivo à amamentação, o que pode contribuir para esses achados 13. É importante destacar que neste estudo um grupo de crianças teve o aleitamento materno exclusivo suspenso durante a hospitalização. Dessa forma, a internação pode ser um potencial confundidor. Além disso, trata-se de uma amostra altamente selecionada, de tamanho pequeno, o que pode ter gerado viés de seleção. 
Este estudo não identificou associação entre a idade materna e o desmame, nem associação com a introdução precoce de outros alimentos, ao contrário dos dados de Oliveira et al. 7, onde se verificou associação entre idade materna e interrupção precoce do aleitamento: filhos de mães menores de vinte anos tiveram maior chance de serem desmamados antes dos seis meses de vida.

Vasconcelos et al. 14 estudaram os fatores associados à duração do aleitamento materno em crianças menores de dois anos, em Pernambuco, e observaram maior duração de aleitamento entre aquelas que residiam na região metropolitana de Recife, cujas famílias apresentavam renda maior que dois salários mínimos e cujas mães haviam freqüentado pelo menos seis consultas de pré-natal. Neste trabalho, não se obteve associação entre a realização de pré-natal e o número de consultas e a idade do desmame, o que pode refletir a pequena ênfase do pré-natal no incentivo à gestante para adoção do aleitamento materno. Nesse estudo, observou-se associação entre a renda familiar e o desmame e também entre a escolaridade materna e o desmame. De forma semelhante, Escobar et al. 15, avaliando crianças atendidas num serviço de emergência da cidade de São Paulo, constataram maior duração do aleitamento materno entre os filhos de mães com maior escolaridade. Resultados semelhantes foram obtidos por Volpini \& Moura ${ }^{16} \mathrm{em}$ um estudo realizado em Campinas, São Paulo. Destaque-se que nossa população de crianças desmamadas foi muito pequena, o que dificulta nossas interpretações.

Esses resultados demonstraram uma introdução mais precoce de leite ou outros alimentos entre as crianças de mães com maior escolaridade, entretanto, um percentual significativo de crianças estava hospitalizado à época da interrupção do aleitamento materno exclusivo, e por isso, essa associação deve ser vista com parcimônia, pois pode estar sendo influenciada por múltiplos fatores ligados à hospitalização. Destaquese, também, o pequeno tamanho da amostra, a população de estudo altamente selecionada e a constatação de que, em geral, trata-se de famílias com baixo nível de escolaridade, não se observando grandes diferenças entre os subgrupos.

Os resultados do estudo revelaram um impacto negativo da hospitalização na amamentação. Esses achados apontam para a necessidade de se adotar medidas para melhorar a assistência pré-natal, sensibilizando as mães e os outros familiares sobre a importância do aleitamento materno. A maternidade deve dar continuidade a esse trabalho, que deve ser estendido a todas as unidades que prestam assistência a lactentes. É fundamental que existam unidades de apoio dirigidas para as mães que se encontram em fase de lactação. É imperativo que os hospitais pediátricos contornem as dificuldades de ordem física e que se faça sensibilização e treinamento permanente dos profissionais envolvidos com a assistência a essas crianças, no sentido de assegurar o aleitamento exclusivo nos primeiros seis meses de vida, enfatizando esta necessidade durante a hospitalização. Há real urgência de ser criada a iniciativa amiga da criança em hospitais e ambulatórios gerais que assistem lactentes, com a criação de normas claras e bem fundamentadas sobre o apoio ao aleitamento materno, assegurando-se, também, a avaliação regular dessas atividades.

\section{Resumo}

Trata-se de estudo do tipo corte transversal que objetivou determinar a prevalência de aleitamento materno em lactentes menores de quatro meses, internados por infecção respiratória em um hospital de ensino de Salvador, Bahia, Brasil e descrever a influência da internação na prática da amamentação. As mães foram entrevistadas e os dados sobre alimentação foram obtidos através de ficha padronizada nas quais são registradas informações sobre as práticas alimentares durante a internação. Entre as 97 crianças incluídas, o aleitamento materno exclusivo foi verificado em $57,1 \%$, sendo interrompido em $35,4 \%$ dos pacientes pelo uso de fórmulas lácteas. A média de duração da internação não se associou à introdução de fórmulas lácteas no hospital. Na análise bivariada, houve associação entre maior escolaridade materna e interrupção mais precoce da amamentação exclusiva e entre renda familiar mais baixa e interrupção mais precoce do aleitamento materno exclusivo. A prevalência de aleitamento materno exclusivo foi baixa e em $35,4 \%$ dos casos foi interrompido durante a internação hospitalar, possivelmente, por dificuldades de ordem física e pelo pequeno apoio da equipe de saúde no estímulo à manutenção da amamentação.

Aleitamento Materno; Desmame; Hospitalização; Lactente; Infecções Respiratórias 


\section{Colaboradores}

A. C. S. Sá e C. M. Bastos participaram da coleta dos dados, interpretação dos resultados e da elaboração do artigo. A. B. Diniz contribuiu na interpretação dos resultados e na elaboração do artigo. C. M. C. Mendes colaborou na interpretação dos resultados e na elaboração e implementação da análise estatística. E. L. Souza participou da elaboração do projeto, da coleta dos dados, da interpretação dos resultados e da elaboração final do artigo. L. R. Silva contribuiu na interpretação dos resultados e na elaboração final do artigo.

\section{Referências}

1. Ministério da Saúde/Organização Pan-Americana da Saúde. Guia alimentar para crianças menores de 2 anos. Brasília: Ministério da Saúde; 2002. (Série AS. Normas e Manuais Técnicos, 107).

2. Gartner LM, Morton J, Lawrence RA, Naylor AJ, O'Hare D, Schanler RJ, et al. Breastfeeding and the use of human milk. Pediatrics 2005; 115:496-506.

3. Ministério da Saúde. Pesquisa da prevalência do aleitamento materno nas capitais e no Distrito Federal. Brasília: Ministério da Saúde; 2001.

4. Vieira GO, Almeida JAG, Silva LR, Cabral VA, Netto PVS. Fatores associados ao aleitamento materno e desmame em Feira de Santana, Bahia. Rev Bras Saúde Matern Infant 2004; 4:143-50.

5. Almeida JA, Novak FR. Amamentação: um híbrido natureza-cultura. J Pediatr (Rio J) 2004; 80:119-25.

6. Lamounier JA. Promoção e incentivo ao aleitamento materno: Iniciativa Hospital Amigo da Criança. J Pediatr (Rio J) 1996; 72:363-8.

7. Oliveira LPM, Assis AMO, Gomes GSS, Prado MS, Barreto ML. Duração do aleitamento materno, regime alimentar e fatores associados segundo condições de vida em Salvador, Bahia, Brasil. Cad Saúde Pública 2005; 21:1519-30.

8. Serra SOA, Scochi CGS. Dificuldades maternas no processo de aleitamento materno de prematuros em uma UTI neonatal. Rev Latinoam Enferm 2004; 12:597-605

9. Quattrone F, Dell Oso MA, Sanchis MC, Du Mortier A. Abandono de la lactancia como consecuencia de la internación pediátrica. Med Infant 1995; 2:97-9.

\section{Agradecimentos}

Fundação de Amparo à Pesquisa do Estado da Bahia (FAPESB, processo PS 01/2005), pelo financiamento parcial do trabalho.
10. Silva LR, Vieira G, Dias CPF, Diniz-Santos DR, Ferraz F, Carneiro G, et al. Conhecimento materno sobre aleitamento: um estudo piloto realizado em Salvador, Bahia visando à elaboração de uma cartilha educativa. Rev Ciênc Méd Biol 2005; 4: 186-93.

11. Vannuchi MTO, Monteiro CA, Réa MF, Andrade SM, Matsuo T. Iniciativa Hospital Amigo da Criança e aleitamento materno em unidade de neonatologia. Rev Saúde Pública 2004; 38:422-8.

12. Mascarenhas MLW, Albernaz EP, Silva MB, Silveira RB. Prevalência de aleitamento materno exclusivo nos 3 primeiros meses de vida e seus determinantes no Sul do Brasil. J Pediatr (Rio J) 2006; 82: 289-94.

13. Vieira GO. A Iniciativa Hospital Amigo da Criança, o aleitamento materno e a mastite lactacional em Feira de Santana [Tese de Doutorado]. Salvador: Faculdade de Medicina, Universidade Federal da Bahia; 2005.

14. Vasconcelos MGL, Lira PIC, Lima MC. Duração e fatores associados ao aleitamento materno em crianças menores de 24 meses de idade no estado de Pernambuco. Rev Bras Saúde Matern Infant 2006; 6:99-105.

15. Escobar AMU, Ogawa AR, Hiratsuka M, Kawashita MY, Teruya PY, Grisi S, et al. Aleitamento materno e condições socioeconômico-culturais: fatores que levam ao desmame precoce. Rev Bras Saúde Matern Infant 2002; 2: 253-61.

16. Volpini CCA, Moura EC. Determinantes do desmame precoce no distrito noroeste de Campinas. Rev Nutr PUCCAMP 2005; 18:311-9.

Recebido em 23/Mai/2007

Versão final reapresentada em 02/Out/2007

Aprovado em 25/Out/2007 